\title{
NOTA PRÉVIA
}

\section{TRYPANOSOMA CRUZI ANTIGENS DETECTED BY IMMUNOELECTRON MICROSCOPY IN THE SPLEEN OF MICE SEROLOGICALLY POSITIVE BUT PARASITOLOGICALLY CURED BY CHEMOTHERAPY. (Preliminary Report).}

\section{S.G. Andrade, L.A.R. Freitas, S. Peyrol, A.R. Pimentel and M. Sadigursky.}

An "immunological memory" has been claimed as responsible for the persistence of serological positive tests in patients with Chagas' disease after being subjected to specific treatment and apparently cured. In mice, negative parasitological tests may coexist with positive specific indirect immunofluorescence test (IIFT) after prolonged treatment with Nifurtimox or Benznidazole 1 . With the objective of investigating whether a lack of correlation between parasitological cure and negative serology is due to the presence of $T$. cruzi antigens bound to the dendritic splenic cells, the present investigation was performed to identify, by immunoelectron microscopy, the presence of $T$. cruzi antigens specifically labeled with peroxidase. With this purpose, mice infected with $T$. cruzi $\left(5 \times 10^{4}\right.$ blood forms) of the $12 \mathrm{SF}$ strain, both in the acute and in the chronic phase of the infection, were subjected to treatment with Benznidazole (100mg k/bw) during 90 days. Parasitological and serological tests were performed 3 to 6 months after the end of treatment. The parasitological tests consisted of haemoculture, xenodiagnosis and subinoculation of the blood into suckling mice. As serological tests the specific indirect immunofluorecence reaction using culture forms as antigens, was used. Investigation of specific antigens in the spleen: sections of the spleen were fixed in paraformaldehyde for immunoelectron microscopy; cryostat sectios $(6$ to $8 \mu \mathrm{m})$ were treated with monospecific purified rabbit anti- $T$. cruz $i$ antibodies and revealed with goat anti-rabbit immunoglobulin bound to peroxidase. As control, sections not treated with the specific anti-serum were used. The labeled tissues were included into Epon resin blocks; ultrathin sections were examined without contrast with a Zeiss EM 109 electron microscope. The following experimental groups were submitted to immunolabeling with peroxidase: I - 10 mice treated and parasitologically negative; II -8 mice treated and parasitologically positive; III -5 untreated infected $\mathrm{c}$ ntrols; $I V-2$ positive controls (acute phase of infectio.); $V_{-}-5$ intact controls. Results: in the acutely infected mice (positive controls), amastigotes

Supported by the UNDP/WORLD BANK/WHO Special Program and Conselho Nacional de Desenvolvimento Científico e Tecnológico - CNPq.

Recebido para publicação em 9/9/87. forms of $T$. cruzi (Figure 1) showed positive immunolabeling with peroxidase on the parasite surface membrane, in the intracellular vacuole membrane and in desintegrated fragments inside macrophages. The specificity of the reaction was controled by the examination of sections without the specific antibodies, which were negative. In the untreated controls as well as in the treated mice (parasitologically positives or negatives) serologically positives by IIFT, it was detected in the germinal centre of folicles of the spleen the presence of positive specific deposits on the membrane of the dendritic cells cytoplasmic processes (Figures 2,3).

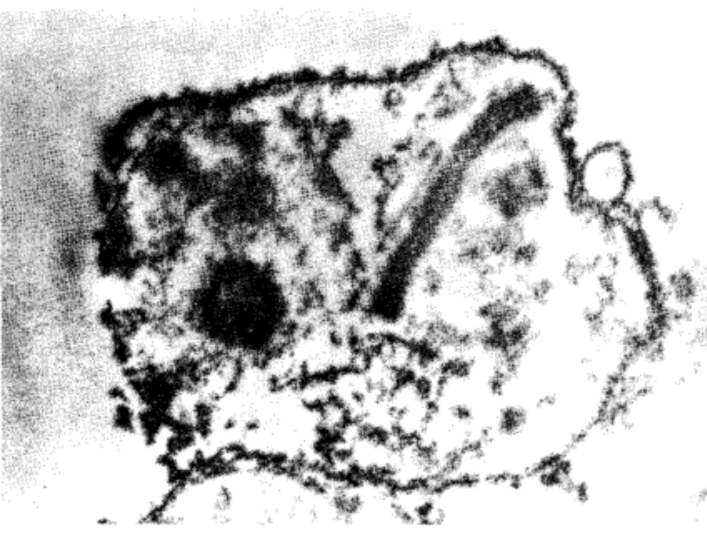

Figure 1 -Amastigote form of $\mathrm{T}$. cruzi in the spleen, showing positive immunolabeling with peroxidase on the parasite surface membrane (Positive control). X 12.000

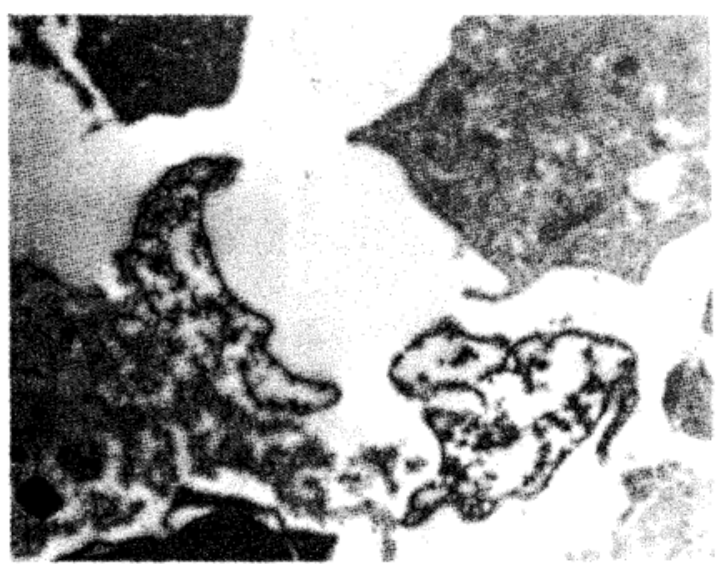


Nota Prévia. Andrade SG, Freiras LAR, Peyrol S, Pimentel AR, Sadigursky M. Trypanosoma cruzi antigens detected by immunoelectron microscopy in the spleen of mice serologically positive but parasitologically cured by chemotherapy (Preliminary Report). Revista da Sociedade Brasileira de Medicina Tropical 21: 41-42, Jan-Mar, 1988

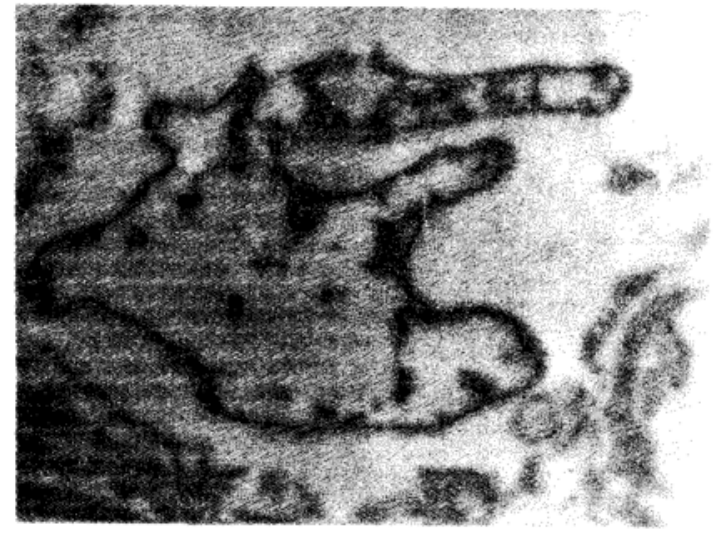

Figura 2 and 3 - Positive specific deposits immunolabeled with peroxidase on the membrane of dendritic cell cytoplasmic processes (treated mice: parasitologically negative and serologically positive). $X 7.000$ and 12.000
Conclusion: It was demonstrated the presence of specific antigens of T. cruzi in treated and parasitologically cured mice, which could be correlated with the presence of an "immunological memory" resisting post-treatment. These results showed the viability of the method for identifications of $T$. cruz $i$ antigen particles in the tissues and open new perspectives for the interpretation of therapeutic results in Chagas' disease.

\section{REFERENCES}

1. Andrade SG, Magalhães JB, Pontes AL. Evaluation of Chemotherapy with Benznidazole and Nifurtimox in mice infected with Trypanosoma cruzi strains of different types. Bulletin of the World Health Organization 63:721-726, 1985. 